lic opinion has a powerful influence, especially the opinion of men. Women dress not for their own comfort, or the health of posterity, but to please their brothers, their lovers, and their husbands. Men could wield a mighty influence for good in this work if they but realized its importance. As man has a more extended field for observation, woman has come naturally to regard his opinions with deference.

Women physicians, especially, work here at a disadvantage. With most women, save those in the advance ranks of thought, any suggestion in the way of hygiene in dress is immediately branded as Dress Reform, and straightway the masculine, pantaloontype of radical dress is brandished and they say, "What else could we expect from one who would study medicine?"

Woman does not like to be laughed at and called strong-minded. She would rather suffer pain and discomfort in her body than to be wounded in her heart by those whom she loves. This seems a plight -certainly we are warranted in using the word, for never was mortal more plicated, enfeebled, almost inextricably entangled-this seems a plight in which you, my brothers, have a work to accomplish, which no one else can do. Will you do it?

\section{LEFT LAPAROTOMY FOLLOWED (A WEEK LATER) BY RIGHT LAPAROTOMY FOR SUPPURATIVE PERITONITIS,}

Consequent upon Bulimia, Fæcal Impaction, Perityphliti, and Septicæmia. Recovery. Also on the Use of Arsenie in Septicæmia.

Read before the District of Columbia Medical Society, Fanu. arv $I T, 1888$.

BY J. F. HARTIGAN, M.D.,

PROFESSOR OF DISEASES OF CHILDREN MEDICAL DEPARTMENT GEORGETOWN UNIVERSITY; ONE OF THE SURGEONS TO PROVIDENCE HOSPITAL, ETC., WASHINGTON, D. C.

I was called on January 24,1887 , to see O. B., æt. I r, the youngest of six children. The mother said he had been complaining for several days of a "stuffy" feeling and headache, and not being in his usual spirits, she thought he needed medicine. Having found upon examination no distinctive symptoms, I prescribed on general principles a brisk cathartic, and gave instructions to inform me in a day or two should he not be better. I was called again on the 26 th, and found that the medicine had acted once, but he continued dull and stupid, not caring to leave his bed. His skin was now hot and dry, tongue furred, pulse accelerated, temperature $100^{\circ}$, and there was tenderness over the ileo-cæcal valve, but no pain, the mischief seeming to point to impacted faeces. Repeated doses of calomel, colocynth, and aloes were ordered, followed by copious injections of castor-oil and turpentine emulsions, without results. A week from the first time I saw him, having contemplated the use of the long tube, Dr. I. W. Bulkley was called in, but upon consultation it was deemed advisable before resorting to it to try once more a large dose of calomel, and accordingly 6 grains were given that night with an equal quantity of bicarbonate soda. This had the desired effect, so that next morning the doctor discontinued his attendance supposing that the difficulty had been overcome.

For several days subsequently there was no appreciable change in the patient; he had no inclination to leave his bed, the amount of fæcal matter that continued to pass being something extraordinary. Contrary to directions he had been given such food as he craved, which was generally of a gross kind, and I also learned for the first time that before his illness he was in the habit of buying a pound of sugar at a time and eating it in the store, or he would go to the butcher's and purchase a like quantity of bologna sausage or hogshead cheese, although he had plenty to eat at home, in fact it was evident that we had to deal with an aggravated case of bulimia and its sequelæ. As I have just stated, through an overindulgent mother and a too-willing servant, our efforts were thwarted in abating resulting perityphlitis. I was bewildered at the copious evacuations and the daily exhibition that confronted me, for it seemed to have been next to a religious duty to save everything for my inspection.

'The first alarming symptom appeared the thira week of his illness, when one morning I found him pulseless at the wrist, skin pale and covered with a cold clammy sweat, eyes dull, breathing imperceptible and gasping. This state of collapse had taken place only an hour before my arrival, and it did not need any intimation to convince the mother-who was the only person present-that her boy was sinking. Having hurriedly given hypoderms of whisky and ordered bottles of hot-water, and mustard to the extremities, I drove off for Dr. Bulkley. On return. ing there was no change, and it seemed that further efforts must be futile. The doctor agreed with me that an abscess had burst into the abdominal cavity, which accounted for the suddenness of the attack, and that apparently the patient had not many hours to live. The treatment was continued, however, and towards evening we were rewarded by commencing reaction. The pulse gradually returned to the wrist, the skin became warm and the breathing more regular; the familiar cry for something to eat was again heard.

But now there was a formidable complication to grapple with. In a few days the patient began to lose flesh rapidly, although his digestion had not failed; he became restless and wakeful, so that hypodermic injections of morphia were the rule. His face presented an anxious look; occasionally as night approached he would become delirious; his tongue was dry and furred. Eight-drop doses of Fowler's solution were now ordered, alternated with 4 grains ferri et quinia citras three time daily, with milk punch and other appropriate nourishment. About the seventh week the parotid glands began to enlarge, and fluctuation being evident in three or four days, I made free incisions, under cocaine, just behind the lobes of both ears, making counter-incisions below the angles of the inferior maxillæ. Having evacuated the contents, amounting to about twelve ounces of putrid-smelling pus, I inserted 
drainage-tubes through upper and lower wounds, and upon their removal within a week all traces of discharge had disappeared-the face resuming its wonted shape instead of the full-moon aspect it had previously borne.

For a week subsequently the lad became brighter, a decided mitigation in the symptoms followed, and there was reasonable ground for belief that the poison was being rapidly eliminated. But the same difficulty continued with his food; protest or argument did not avail-even a threat to abandon the case-there was always some one to bribe, or bring him a banana or pancake, or whatever there was in the kitchen, and the usual message would come for me at night to give him a corrective, or hypodermic of morphia and atropia to stem the resulting mischief. Even the collapse-which I argued was brought about in this way-did not deter the servant from continuing her pig-headed course in response to his perpetual cravings.

Towards the end of March another crisis occurred; the temperature rose to $103^{\circ}$, pulse was small and fluttering, skin dry, face ashy, with an anxious, drawn look, urine scanty, tongue brown, thick sordes about the teeth. In a few days the body shrunk remarkably, and an extensive bed-sore appeared, through which the posterior superior spinous processes of ilii and middle segment of the sacrum almost extruded. Another development about this time, which contributed to the rapid emaciation, was the vomiting of green fluid streaked with mucus; the amount per day for two weeks averaged one pint, which was reserved for my inspection as religiously as the enormous quantities of fæcal matter passed in the first weeks of his illness. There seemed to be no effort required to eject this fluid; he would call feebly or point for his basin; and apparently would vomit with the same ease that a suckling infant sometime regurgitates its milk.

When the case appeared hopeless and all our efforts to save the patient were in vain, my attention was attracted to a suspicious fulness in the left iliac region. Dr. Bulkley examined it with me next morning, and both agreed that it was pus. The propriety of laparotomy at once suggested itself. Was it advisable to do it in the face of almost certain dissolution? The mother, having been approached on the subject, left the matter with me, notwithstanding the hue and cry raised by some of the relatives and neighbors that it was cruel to operate on a dying child. The matter was discussed by the doctor and myself, and it is only justice to him to say that he did not entirely approve it-adding that I would be condemned if the child should die on the table, as was extremely probable. Seeing that there was no hope otherwise, and having the mother's consent, I held that it was an unwritten law in surgery not to let a patient die for want of an operation; so at my request the doctor consented to be present.

Accordingly, the same afternoon, having administered a full dose of stimulant, Dr. C. J. Osmun giving the ether, I made an incision three inches long over the most prominent part of the swelling. When about entering the peritoneum, which was soft and congested, the pulse could not be detected and the breathing was hardly perceptible. While the ether was suspended and the usual artificial means were being employed to resuscitate him I completed the operation, giving exit to more than a pint of foulsmelling pus. This portion of the abdominal cavity having been thoroughly cleansed, the edges of the wound including the peritoneum were drawn together and sutured, and a drainage tube inserted. In a little while reaction was established, the skin became warm, and the pulse and breathing stronger. On visiting my patient later in the evening his condition continued to improve. He said they had not given him enough to eat since he saw me-only one chop and a couple of rolls!-and it was explained for my edification that it had not hurt him, notwithstanding the positive instructions that he should have nothing but milk punch and beef extract. Having given the usual hypodermic I left him for the night.

Next morning he was bright and cheerful, after a prolonged sleep; his bowels moved freely. Ordered balsam of Peru and iodoform to be applied to the bedsore, instead of flaxseed poulticing, which threatened sloughing. Fowler's solution and the citrate of iron and quinine were continued. For several days the patient steadily gained ground, the wound discharged healthy pus abundantly, the emaciated limbs and thorax and shrunken face began to fill up, and there was an improved appearance of the bedsore.

In a week, however, all was changed by a recurrence of dangerous symptoms; the temperature again rose, there was jactation, delirium, hectic, brown tongue, slight rigors, hot and dry skin. On the right side of the abdomen, corresponding to the situation in the left, was noticed another enlargement more diffuse and fluctuating. The chances of objection to a second operation being removed by the success of the first, the next day, with Drs. Bulkley and Osmun, I made a similar incision, under ether. No alarming incident occurred during the operation, which was similarly performed and which resulted in the evacuation of nearly another pint of pus; the cavity was washed out and sponged thoroughly, with antiseptic precautions, and the wound dressed after the method of the first. During the night all urgent symptoms disappeared, but the boy now showed great exhaustion. Under the judicious use of stimulants, the continuance of his medicine, and greater care with his food, he gradually merged into a state of convalescence. Both wounds were dressed daily, any retained matter being gently pressed out; as the discharge diminished they filled up, so that two weeks after the first operation one drainage tube was removed, the other following a few days later.

All anxiety was now over; the boy steadily gained flesh and strength, bedsore healed, the tongue became clean, pulse and temperature normal-more than that his appetite became normal-so that about the Ist of May he was able to sit up. He called at my office on the 18 th of May in good health, and is to-day a strong, vigorous boy.

REMARKS. - The foregoing case is unique in many respects, and is offered as a contribution towards the establishment of surgical procedure in a condition in 
which the patient has heretofore been abandoned to his fate. Richter suggested more than a hundred years ago that if in the abdominal cavity milk-like fluids are formed, operation is the only remedy for removal of the disease. Whether he meant the more formidable one of laparotomy, or puncture by the trocar, does not appear; but it is certain that the latter method only has been employed until a comparatively recent date-modern surgeons, being dissatisfied with the results thus obtained, preferring laparotomy.

The first recorded case that I can find is by Bertels in $187 \mathrm{I}$, who made a 2 -inch incision in the abdomen of a supposed phthisical patient, and successfully removed two wash-basins full of pus. Then followed Boye, Studensky, Krönlein, Selmer, Tait, Elias, Barwell, Marsh, Caselli, Schmidt, Roberts and otherspioneers who have placed this among the accepted operations in surgery. The cases reported are principally for perforation or consecutive peritonitis from rupture of some portion of primæ via, bursting of a pyosalpinx or pelvic abscess, Mr. Tait presenting by far the largest percentage of recoveries; but I find none with a history similar to my own, viz.: the depraved appetite, impaction of fæces, perityphlitis, suppuration of the parotid glands as well as the peritoneum following septicæmia-besides the dietetic errors during treatment, which were so exasperating.

With regard to diagnosis in these cases, it is remarkable how often errors are made by men of large experience; errors which will probably be continued and, except in rare cases, be deemed excusable. have at present on hand an illustration in which there is a history of pelvic abscess of more than two years' standing, with anchylosis of hip, following a miscarriage. Having been sent to a hospital in this city last spring for treatment, the patient was discharged by the surgeon with the statement that the institution was for the treatment of diseases peculiar to the sex, and that there was nothing the matter with her but hip-joint disease. At that time the same condition undoubtedly existed (at least in a less degree) which a month ago led to an incision by myself in the left dorsum ilii, giving exit to a large quantity of pus, followed since by discharge of fæcal matter. The doctor, however, is in good company, for during the late International Congress an eminent London obstetrician saw the patient and gave directions how the anchylosed hip might be overcome, losing sight of the fact that there was pelvic abscess with fistulous communication in the rectum.

The interesting question in these cases is when to operate, or when an exploratory incision should be made. I think Dr. R. S. Sutton reflects the advanced sentiment of the profession, in this respect, in the following terse paragraph:

"In short, those who have acquainted themselves thoroughly with this subject, from a clinical standpoint, are ready to open the wall of the abdomen in any case where death threatens from any cause evidently amenable to surgical procedure, or any cause which is obscure, and which can be only understood after the section is made. In many instances it is substituting an ante-mortem for a post-mortem ex- amination. The difference to the patient is, that recovery and cure will often follow the ante-mortem examination, but recovery has never been known to follow the post-mortem examination."

Let me call attention briefly to what I consider the points of interest in my case. It may first be asked why abdominal section was not made earlier in the disease; for instance, shortly after the collapse of the third week? Of course it could not have been done at the time of collapse; and, strangely enough, when reaction followed from the supposed bursting of the abscess, there were no symptoms to justify such a course, nor were there previously, as the abdomen was flat and quite tolerant of pressure. I think the Society will agree with me that the fact that the boy rallied so well, and was free from pain and chills, as well as distension of the abdomen, precluded the propriety of such interference, and that if it had been done, and death had followed, we would not have been able to present such an interesting statement this evening.

It may be instructive to account for the green fluid vomited, the persistence and quantity of which, while it lasted, was so amazing. Of the roo or more cases consulted, going back forty years, in which the trocar was the principal agent employed in evacuating the pus, in only two or three has this been noted as a symptom. There was costiveness and digestive disturbance frequently. Perhaps peristalsis is impeded in consequence of the effusion; the antiperistaltic motion, on the other hand, being increased, thereby produces nausea and vomiting.

The last point to which I desire to call attention is the use of arsenic in septicæmia. While a subject of this disease myself from a dissection wound about fifteen years ago, I was found roaming around aimlessly, half delirious, with my right arm seemingly twice its size, glazed and tender to the touch, and feeling like a leaden weight. I saw Dr. Schafhirt, Anatomist of the Army Medical Museum, who advised Fowler's solution in Io drop doses every 2 or 3 hours, and free stimulation. Before morning the change was remarkable: fever and threatening symptoms had disappeared, and I steadily gained ground, so that within three days the odema had subsided without developing into suppuration. Having had several dissection wounds since, I have always as a matter of precaution taken arsenic for about forty-eight hours, without any untoward symptoms following.

I am reminded, in this connection, of a case of acute suppuration of the knee-joint, with septicæmia, admitted to Providence Hospital about two years ago. The œdema and suppuration of the thigh and leg were very considerable and extended above the hip, presenting an erysipelatous appearance.

The following is a synopsis of the case furnished me by Dr. D. P. Hickling, the then house physician, who said that as one of a similar character had died a short time before during his service, in spite of the most active treatment, and this seeming to be the worst, the prognosis was, to say the least, discouraging:

"W. C., æt. 22, white, male, entered Providence Hospital January 21, 1886, giving the following his- 
tory: On the morning of January 15 , while wheeling a barrow up an inclined board, he slipped and fell to the ground heavily upon his right knee, striking a stick or stubble which caused a punctured wound of the knee. He had suffered considerably since, but had not been confined to bed. When seen his condition was as follows: Tongue coated, skin dry and hot, with general prostration and considerable pain, redness and swelling in joint, extending to the hip. A brisk cathartic was ordered, and opium at bedtime, also potass. nitras for fever. Milk punch, quinine and tinct. ferri chlor. were given liberally, and opium was continued to relieve pain.

"January 24 , evening temperature was $102.4^{\circ}$, pulse 106, tongue heavily furred, symptoms of great prostration; wound discharging freely, and general redness of leg and thigh with excruciating pain. Dr. Hartigan made several incisions for more thorough drainage of the joint, and called a consultation for the following day.

"January 25. Morning temp. $100^{\circ}$, pulse roo; evening temp. 10r. $4^{\circ}$, pulse roo. Prostration still great, and for constipation brisk cathartic ordered. Consultation decided that no advantage could accrue from any operation, and suggested continuance of the treatment, and thorough drainage of joint.

"January 26. Temp. (morning) $99.4^{\circ}$, pulse 98 . Evening temp. Iоr. $6^{\circ}$, pulse 94 .

"January 27 . Morning temp. $99.8^{\circ}$, pulse 92 ; even. ing temp. $102^{\circ}$, pulse roz.

"January 28. Morning temp. 98.8 , pulse 100 ; evening temp. $100.4^{\circ}$, pulse roo.

"January 29. Morning temp. 99.4 ${ }^{\circ}$, pulse I04; evening temp. $102^{\circ}$, pulse 94 . Patient's limb has been thoroughly drained under ether by numerous incisions, and washed daily with solution of bromine comp. In spite of the most stimulating treatment and nourishing diet, his general condition was critical-seemed to be getting worse every day, when Dr. H. ordered I 2 drop doses of Fowler's solution three times daily.

"January 30. Morning temp. $99.6^{\circ}$, pulse 98 ; evening temp. $100^{\circ}$, pulse 96 -the lowest evening temperature since aćmission.

"January 3 r. Morning temp. $99^{\circ}$, pulse 98 ; evening temp. roo. $8^{\circ}$, pulse 98 . Fowler's solution increased to 20 drops three times daily.

"For two or three weeks subsequently patient's condition did not vary much, except a few evenings when the temperature rose to $103^{\circ}$, and further drainage was necessitated. Dr. H. made altogether sixteen incisions from time to time, averaging from 3 to 6 inches long, with drainage-tubes of different sizes intercommunicating; the pus burrowed between the sheaths of the muscles of thigh and leg close to the bones, and a strong solution of chloride of zinc was substituted for the bromine and injected through the sinuses.

"After the middle of February the patient commenced steadily to improve, although occasionally there was a temporary rise of temperature. He remained in the hospital until the first week of April, when he was discharged cured, with a useful limb, and no anchylosis of the knee-joint."
I could mention other cases of septicæmia, both in hospital and private practice, where Fowler's solution was the chief treatment, with almost uniform success; but I will not longer detain the Society. I0- or I 2-drop doses every four hours are not excessive untii the temperature diminishes, or other symptoms improve, gradually reducing according to indications.

I believe, therefore, that, in closing, I am justified in saying that in all cases of septicæmia, or wherever there is pus, free drainage and arsenic are the keynotes to success.

\section{CASES OF ABDOMINAL SURGERY.}

1. Abscess of Liver; Incision and Drainage-Reeovery. 2. Epithelioma of Esophagus; Gastrostomy-Death. 3. Large Uterine Fibroid: Exploratory Incision-Recovery. 4. Strangulated Hernia; Resection of Intestine-Recovery. 5. Ruptured Ovarian Cyst; Ovariotomy-Death. 6. Encysted Pelvic Abscess-Recovery. 7. Double Ovariotomy-Recovery.

Read before the Philadelphia County Medical Society, April II, 1888

BY J. M. BARTON, M.D., OF PHILADELPHIA.

By invitation of your Board of Directors I submit some specimens, this evening, from cases of abdominal surgery and present the following notes for your consideration:

Case 1.-Abscess of liver. Free incision and drainage; recovery. - George B., at. $3^{8}$ years, was admitted to the medical wards of the Jefferson Medical College Hospital, July 29, under the care of my colleague, Dr. Neff. The patient was suffering with an immense abscess of the liver, extending the area of the percussion dulness to below the umbilicus and to the left of it. At the request of Dr. Neff, I removed by aspiration more than a quart of "brickdust" colored pus, with such relief that the patient was able to return to his home in the interior of the State. The abscess cavity rapidly refilled, and he returned to the hospital, when we decided to operate by the method of Dr. Ransohoff, of Cincinnati. Making an incision through the abdominal wall, five inches in length, at the outer edge of the right rectus nuuscle, permitting it to gap, fastening the edges of the wound by sutures to the liver, and when firm adhesions had taken place, opening the liver by the galvanic knife. When adhesions were found to have formed, and I attempted to divide the tissues of the liver with the galvanic knife it did not act well; at first, while white-hot, it would cut readily, but the resulting very free bleeding quickly short-circuited the current and the knife became instantly cold. After repeated trials it still proved so unsatisfactory that an ordinary scalpel was substituted, with which the pus cavity was reached. An attempt to check the bleeding from the margins of the incision, by the cautery knife, was also unsuccessful, and it was only 